\title{
Temporomandibular Joint Ankylosis with incidental findings of Odontogenic keratocyst and Mucous Retention Cyst: Report of a Case
}

\author{
Dr. Pawan Motghare ${ }^{1}$, Dr. Aarti Bedia ${ }^{2}$, Dr. Sumit Bedia ${ }^{3}$, \\ Dr. Sangeeta Bhattacharya ${ }^{4}$ \\ ${ }^{I}$ (Assistant Professor, Department of Oral Medicine \& Radiology, Vidya Shikshan Prasarak Mandal's Dental \\ college \& Research centre, Nagpur/ Maharashtra university of health sciences,Nashik, India) \\ ${ }^{2}$ (Assistant Professor, Department of Oral Medicine \& Radiology, Bharati Vidyapeeth Dental College \& \\ Hospital, Mumbai/ Bharati Vidyapeeth deemed university, Pune, India) \\ ${ }^{3}$ (Reader, Department of Prosthodontics, Bharati Vidyapeeth Dental College \& Hospital, Mumbai/ \\ Bharati Vidyapeeth deemed university, Pune, India) \\ ${ }^{4}$ (Lecturer, Department of Oral Medicine \& Radiology, Vidya Shikshan Prasarak Mandal's Dental college \& \\ Research centre, Nagpur/ Maharashtra university of health sciences,Nashik, India)
}

\begin{abstract}
Temporomandibular joint ankylosis is inability to open mouth due to either a fibrous or bony union between the head of the condyle and the glenoid fossa. It is most commonly associated with trauma, local or systemic infection, tumors or systemic disease such as ankylosing spondylitis, rheumatoid arthritis, or psoriasis. Odontogenic keratocyst as defined by World Health Organization are known for their peculiar behavior, varied origin, debated development, unique tendency to recur, and disputed treatment modalities. The prevalence of mucosal retention cysts in the maxillary sinus varies considerably from report to report, perhaps as a function of population, geography and season. The detection and correct interpretation of the retention cyst is important for preventing unnecessary diagnostic procedures or surgical intervention. We report a case of temporomandibular joint ankylosis with incidental finding of odontogenic keratocyst and mucous retention cyst.

Keywords - Temporomandibular joint ankylosis, Odontogenic keratocyst, Mucous Retention Cyst
\end{abstract}

\section{INTRODUCTION}

Temporomandibular joint ankylosis: Temporomandibular joint (TMJ) ankylosis is a disorder that leads to a restriction of the mouth opening from partial reduction to complete immobility of the jaw. It is most commonly associated with trauma, local or systemic infection, tumors or systemic disease, such as ankylosing spondylitis, rheumatoid arthritis, or psoriasis. TMJ ankylosis may be classified by a combination of location (intra- or extra-articular), type of tissue involved (bony, fibrous, or fibro-osseus) and extent of fusion (complete, incomplete). Literature classifies ankylosis as true and false. Any condition that gives rise to osseous or fibrous adhesion between the surfaces of the TMJ is a true ankylosis. False ankylosis results from pathologic conditions not directly related to the joint. [1]

The impairments on orofacial function included; limited chewing ability, alterations in speech, compromised oral hygiene and dental care, restricted airway, and decreased posterior dental eruption. The clinical features of unilateral TMJ ankylosis include; facial asymmetry, chin deviation to the affected side, elongation and flatness on the nonaffected side with roundness or fullness on the affected side when observed from a frontal view. A bony thickening is often felt in the preauricular area of the affected TMJ. The lateral profile has a reduced mandibular projection with "bird face deformity" in the most severe cases. The degree of mandibular recession depends on the severity, unilateral or bilateral joint involvement, age of onset, and duration of TMJ ankylosis. The mandibular morphology is severely influenced in terms of size and shape with marked antegonial notch, enlarged coronoid process, reduced vertical ramus height on the affected side; and flattened mandibular body and ramus on the non-affected side. The ankylosed mandibular condyle can be hyperplasic with irregular contours and absent joint spaces. [2]

The first goal in the management of TMJ ankylosis is the resection of the ankylosed segment and reestablishment of the mandibular movement. Additional surgical procedures are required for the reconstruction of the TMJ and atrophic ramus. A number of surgical techniques for purpose have been reported, such as gap arthroplasty or interpositional arthroplasty, and reconstruction with costachondral graft, total joint prosthesis, free vascularized bone or total joint graft. [3] Fibrous ankylosis may be treated by functional method. [4]

Odontogenic keratocyst: The odontogenic keratocyst (OKC) was first described in 1876 and named by Phillipsen in 1956. It is one of the most aggressive odontogenic cysts of the oral cavity. OKC is 
known for its rapid growth and its tendency to invade the adjacent tissues including bone. It has a high recurrence rate and is associated with the basal cell nevus Syndrome. OKC are generally thought to be derived from either the epithelial remnants of the tooth germ, or the basal cell layer of the surface epithelium. The majority of patients are in the age ranges of 20-29 and 40-59, but cases ranging from 5 to 80 years have been reported. In one study the average age of males was 9.7 years older than that of females. The distribution between sexes varies from equality to a male to female ratio of 1.6:1, except in children. OKC may occur in any part of the upper and lower jaw with the majority occurring in the mandible, most commonly in the angle of the mandible and ramus.

Radiographically, OKC present predominantly as a unilocular radiolucency with well-developed sclerotic borders. They may also present as a multilocular radiolucency. These lesions grow to sizes larger than any other odontogenic cyst. They more often penetrate the bone rather than expand it and grow in an anterior to posterior direction. Despite this aggressive growth, they often remain asymptomatic.

$\mathrm{OKC}$ are significant clinical entities due to their tendency for recurrence and aggressive behavior. These cysts are known to have a high recurrence rate ranging between 13 to $60 \%$. Complete surgical removal is the treatment of choice. Other investigators advocate marsupialization and occasionally resection for the more aggressive cysts that tend to perforate buccal and lingual bone. Resection is a rare modality of treatment. onservative surgical removal and long-term follow-up is the treatment of choice by most clinicians. [5]

Mucous retention cyst of maxillary antrum: The actual pathogenesis of these lesions is controversial. One etiology suggests that blockage of the secretary ducts of seromucous glands in the sinus mucosa may result in pathologic submucosal accumulation of secretion, resulting in swelling of the tissue. A second possibility suggests that the serous nonsecretary retention cyst arises as a result of cystic degeneration within an inflamed, thickened sinus lining. The retention cyst rarely causes any signs or symptoms and thus the patient is usually unaware of the lesion. It is often noticed as an incidental finding on radiograph made for other purposes. Radiographically it appears as noncorticated, smooth, dome shaped radiopaque mass. Maxillary sinus is most common site of antral retention cyst. [6]

The prevalence of mucosal retention cysts in the maxillary sinus averages around 5\%, but varies considerably from report to report, perhaps as a function of population, geography and season. The prevalence is approximately twice as high in men as in women. The detection and correct interpretation of the retention cyst is important for preventing unnecessary diagnostic procedures or surgical intervention. [7] Mucosal retention cysts in maxillary sinus usually require no treatment because they customarily resolve spontaneously without any residual effect on the antral mucosa. [6]

\section{CASE REPORT}

A 20-year-old female patient (Fig 1) visited to Department of Oral Medicine and Radiology, Sharad Pawar Dental College, Sawangi (M) Wardha, with chief complaint of inability to open mouth since one and half months. Patient's history revealed that she had an episode of trauma to her chin due to a trivial fall during play time at age of 12 years. She developed swelling infront of right ear subsequent to trauma, which resolved in due course of time. After resolution of swelling her mouth opening started reducing gradually over a period of 7 years. Patient was on liquid diet since one month.

On examination her facial symmetry was asymmetrical due to flattening on left side of face and slight deviation of mandible on right side. Lips were incompetent. Right condyle was not palpable. Her mouth opening was nil. Antegonial notch was prominent on right side.

A panoramic radiograph revealed osseous proliferation in right TMJ obliterating the joint space and right sigmoid notch involving right coronoid process suggestive of bony ankylosis of right TMJ (Fig 2).

Two incidental findings were seen on same panoramic radiograph when observed carefully. One is single unilocular well defined pericoronal radiolucency with hyperostotic borders encircling the entire crown of 47 suggestive of dentigerous cyst with 47(Fig 3). Other is single well defined semicircular dome shaped radiopacity of uniform density seen extending from lateral wall of left maxillary antrum into the antrum itself forming dome shaped radiopacity suggestive of mucous retention cyst in left maxillary antrum.

To rule out any other pathology in maxillary antrum of left side, water's view was advised which revealed no other findings apart from mucous retention cyst (Fig 4).

After complete evaluation, release of ankylotic mass, gap arthroplasty, right and left side coronoidectomy and interposition with temporalis muscle followed by enucleation of dentigerous cyst in relation with 47 was decided under general anesthesia.

Under all aseptic precaution and condition and standard patient preparation, fibre - optic nasoendotracheal intubation was done. Alkayat and Bramley incision was employed (Fig 5). A $45^{\circ}$ incision given over zygomatic arch region and the arch and ankylotic mass was exposed (Fig 6). $1.5 \mathrm{~cm}$ gap was created by removing the osseous tissue along with coronoid process of right side with surgical burs and chisels (Fig 
7).34 mm mouth opening was achieved (Fig 8). A "U-shaped" axial composite flap (fascia, muscle, and periosteum) was obtained from the exposed temporal muscle and rotated inferiorly under the zygomatic arch through the space created by the osteotomy. The flap was sutured medially, anteriorly, and posteriorly with 3-O Vicryl.

Contralateral coronoidectomy was performed after giving incision over anterior border of ramus of mandible on left side. Temporal flap was inferiorly rotated under the zygomatic arch. A maximum incisal opening of $50 \mathrm{~mm}$ was obtained after the osteotomies.

Dentigerous cyst with 47 was enucleated along with the lining and was sent for histopathologic examination (Fig 9). Intraoral closure was done with 3-O Vicryl and extraoral incision closure done in two layers with 3-O Vicryl and 5-O and 3-O silk.

The post-operative course was uneventful. Post-operative antibiotics and pain medication was prescribed. There was no motor deficit on either side of the face.

Vigorous post-operative physiotherapy was performed to maintain the mobility and to prevent hypomobility secondary to fibrous adhesions. The patient was followed at three months interval, with a maximum mouth opening of $50 \mathrm{~mm}$ (Fig 10). A panoramic radiograph showed no signs of recurrence after 6 months follow up (Fig $11 \& 12$ ).

As mucosal retention cysts resolve spontaneously without any residual effect on the antral mucosa, it was left untreated for follow up radiographic examination.

The histopathologic report of enucleated cyst revealed orthokeratinized stratified squamous epithelium lining with non polarity of basal cells. This lining is covered with connective tissue wall which consist of collagen fibers, fibroblasts and capillaries. Cystic cavity consists of keratin flecks. Thus histopathologic findings were suggestive of Odontogenic Keratocyst with 47 (Fig 13).

\section{DISCUSSION}

Moreover in case of TMJ ankylosis, an appropriate worldwide accepted protocol is to be administered which includes surgical intervention, elaborate resection early mobilization and aggressive physiotherapy for atleast 6 months to one year postoperatively.

It is said that a child learns to explore the world through his mouth! Any pathology that afflicts the TMJ and restricts the mouth opening carries a mental stigma that overweighs the physical disability posed by the problem in growing children. Speech aberrancy, poor oral hygiene, rampant caries and behavioral problem pose unique challenge to dentist. [8] In the present case both the patient and her parents were provided comprehensive psychological rehabilitation before, during and after the surgical intervention.

Early aggressive postoperative physiotherapy has been recognized as an essential for the prevention or treatment of TMJ hypomobility or ankylosis. The biological and physiological basis for increasing the range of motion using dynamic exercise in restoring normal functions after surgery and prolonged immobilization has been well documented in trauma, orthopedic and physical therapy literature. The potential benefits of TMJ opening and closing exercises are improved muscle vascularity, increased muscle mass and protein metabolism, decreased muscle fatigue and increased strength, reversal of the atrophic and degenerative changes within the joints and restoration of the normal internal fibrous structure anatomy. [9]

Interpositional Gap Arthroplasty is a highly effective and safe surgical management option for TMJ ankylosis with acceptable immediate and long term outcome, particularly when temporalis fascia and muscle are used for adults and costochondral grafts with fascia interposition used for children. [10]

A 7-step protocol has been developed for the treatment of TMJ ankylosis: 1) aggressive resection of the ankylotic segment, 2) ipsilateral coronoidectomy, 3) contralateral coronoidectomy when necessary, 4) lining of the joint with temporalis fascia or cartilage, 5) reconstruction of the ramus with a costochondral grafts 6) rigid fixation of the graft and 7) early mobilization and aggressive physiotherapy. [11]

This protocol formed the basis of the treatment plan that was undertaken in this patient, except the reconstruction and rigid fixation of the graft.

The most characteristic clinical aspect of OKCs is the high frequency of recurrence. Since the lining of the $\mathrm{OKC}$ is thin and friable, removal of cyst in one piece may sometimes be difficult. Complete removal of the cyst lining without leaving behind remnants attached to the soft tissue or bone is necessary to avoid recurrence. [12]

Before surgery, the precise extent of the mandibular cysts should be known, as well as its relation to the neighboring anatomic structures, such as the mandibular neurovascular bundle. With an exact knowledge of the buccolingual position of the mandibular canal and cortical bone involvement, the surgical result may be improved. It has been reported that the OKC has different growth characteristics from other odontogenic cysts. Because of its rapid growth and high recurrence rate, preoperative differential diagnosis is important. [13]

Because of the high percentage of recurrence, various methods of treatment are recommended such as marsupialisation, cryotherapy, enucleation with or without the use of additional chemical means/agents and 
enucleation with postoperative intraoral suction and segmental resection of the jaw. [14] The propensity of the OKC to the retromolar area of the mandible is well documented in the literature. [15]

More recent research has shown that differences in proliferative activity in OKCs suggest an alteration of the cell's cycle control producing an increase in cell proliferation that could explain the biological behavior of OKCs. Successful treatment of OKCs is usually attributed to the use of techniques which address the margins of the initially presenting cyst, with the objective of removing all of the epithelial lining. [16]

Post-operative follow-up with annual radiological examinations is essential for at least five years following surgery. Recurrence is documented even after 10 years of follow-up and treatment. [17] In our case patient was advised for follow up check up at an interval of every 3 months for at least 5 years followed by 6 months interval check up for next five years. In our case follow-up radiological examination was planned for mucosal retention cysts in maxillary sinus.

\section{CONCLUSION}

In such cases where multiple pathologic findings are observed, a well planned treatment is to be performed following all basic principles of treatment. A group of findings in same patient does not rule out possibility of syndrome or the reason of one or more incidental findings can be because of the effect of the present pathology. Proper case study, advanced investigations and basic principle of surgery with periodic follow up are key to manage such pathologies.

\section{REFERENCES}

[1] Belmiro Cavalcanti Do Egito V, Ricardo V Bessa-Nogueira, Rafael VC. Treatment of temporomandibular joint ankylosis by Gap arthroplasty. Med Oral Patol Oral Cir Bucal 2006; 11: E 66-69.

[2] Wen-Ching E Ko, Chiung-Shing H, Yu-Ray C, Alvaro AF. Cephalometric craniofacial characteristics in patients with temporomandibular joint ankylosis. Chang Gung Med J 2005; 28: 456-466.

[3] Reha SK, Dogan D, Hakan HT. Reconstruction of temporomandibular joint using distraction osteogenesis: A Case Report. Turk J Med Sci 2001; 31: 569-572.

[4] Shafer WG, Hine MK, Levy BM, Tomich CE.A text book of oral pathology. $4^{\text {th }}$ edn.Philadelphia: W.B.Saunders Company, 1993:705707.

[5] Dolphine O, Valiente R, N. Ghanee. EA. Kenny, Dawson KH. Odontogenic Keratocyst: The Northwestern USA Experience. The J Contemp Dent Pract 2000; 1:1-10.

[6] White SC, Pharaoh MJ. Oral radiology: Principles and Interpretation. $5^{\text {th }}$ edn. St Louis: Mosby, 2004:581-582.

[7] Farman AG, Nortje CJ. Pathologic conditions of the maxillary sinus. Panoramic imaging news 2002; Volume 2, Issue 3. Panoramic Corporation (07-02).

[8] Shashukiran ND, Reddy SVV, Patil R. Yavagal C. Management of temperomandibular joint ankylosis in growing children. J Indian Soc Pedo Prev Dent 2005; 35-37.

[9] Chun-Li L, Yu-Chan K, Lun-Jou L. Design, Manufacture and clinical evaluation of a new TMJ exerciser. Biomed Eng Appl Basis Comm 2005; 17: 135-140.

[10] Iram A, Muhammad J, Muhammad J, Shah MG. Temporomandibular joint ankylosis: Experience with interpositional gap arthroplasty at Ayub Medical College. Abbottabad. J Ayub Med Coll Abbottabad 2005; 17: 67-69.

[11] Bob R, Leland RM. Treatment of temporomandibular joint ankylosis: A case report. J Can Dent Assoc 2001; 67:659-63.

[12] Banu G K, Beliratalay, Mehmt AE. Odontogenic cysts: A clinical study of 90 cases. J Oral Sci 2004; 46:253-257.

[13] Bong-Hae C. Radiographic analysis of odontogenic cysts showing displacement of the mandibular canal. Imaging Sci Dent 2003; 33: 211-5.

[14] Klara S, Jaksa G, Pavel K, Josko G. Surgical treatment of odontogenic keratocysts by intraoral postoperative suction. Acta Stomatol Croat 2003; 37: 105-108.

[15] Nartey NO, Saini T. Odontogenic keratocyst radiographic features. Saudi Dent J 1990; 2:15-20.

[16] Philip JB, David H, Carlos M, Tyler P. The multifocal nature of odontogenic keratocysts. J Calif Dent Assoc 2005; 33:961-965.

[17] Kerim O, Berkay TS, Metin S. A large odontogenic keratocyst containing a third molar tooth in the maxillary antrum. Turk $J$ Med Sci $2005 ; 35: 341-346$.

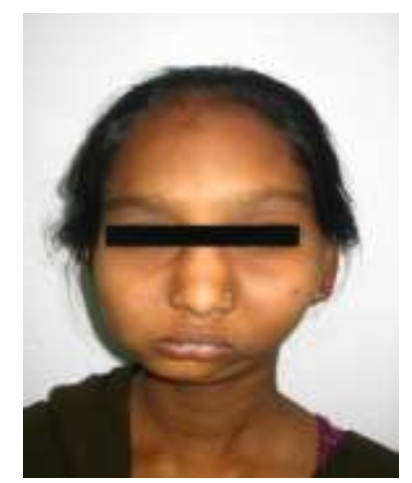

Figure 1: Preoperative photograph of patient with ankylosis. 


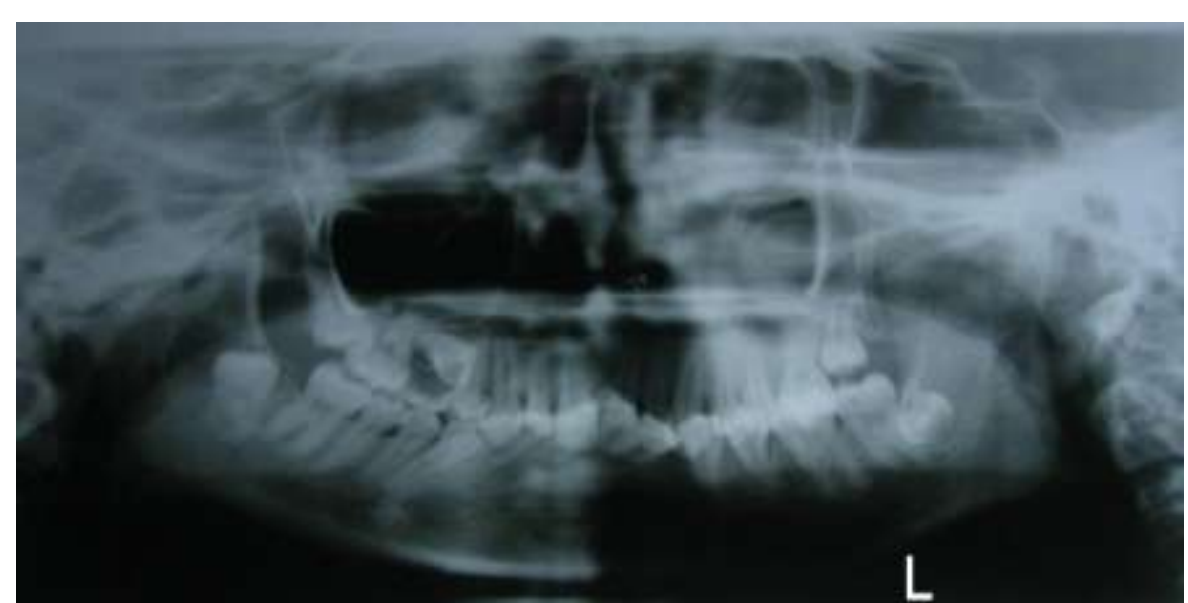

Figure 2: Preoperative orthopantomogram showing bony ankylosis of right TMJ and two incidental findings of dentigerous cyst with 47 and mucous retention cyst in left maxillary antrum.

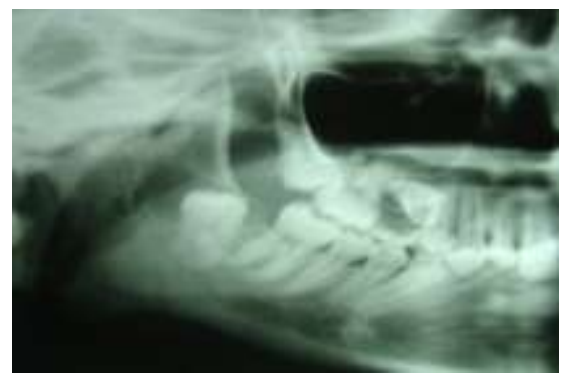

Figure 3: Cropped photograph of orthopantomogram showing bony ankylosis of right TMJ and dentigerous cyst with 47.

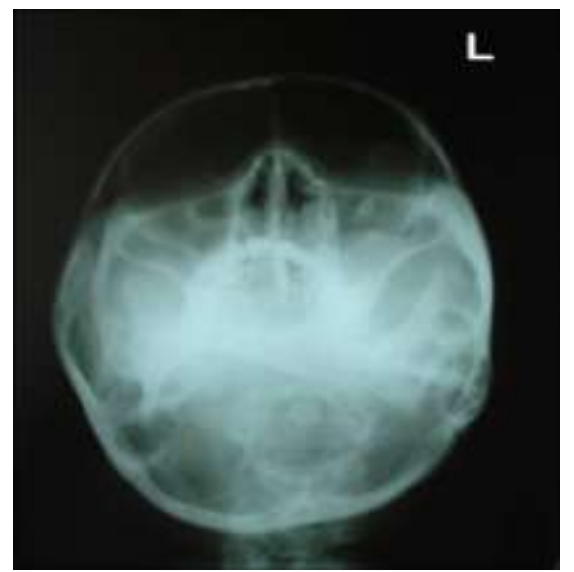

Figure 4: Water's view showing mucous retention cyst in left maxillary antrum.

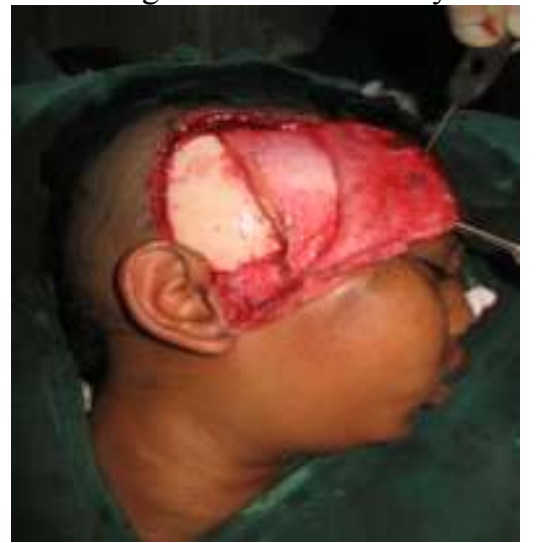

Figure 5: Alkayat and Bramley incision 


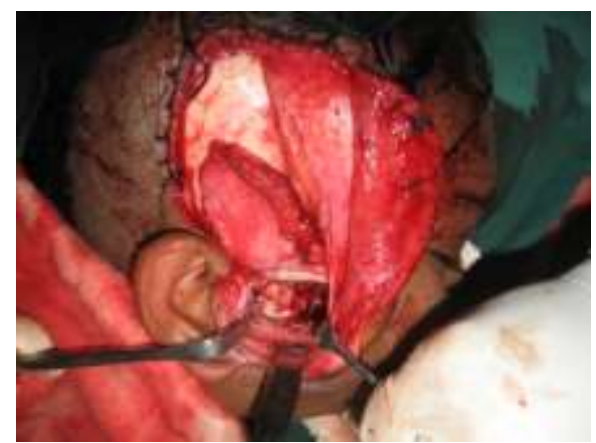

Figure 6: Exposed ankylotic mass.

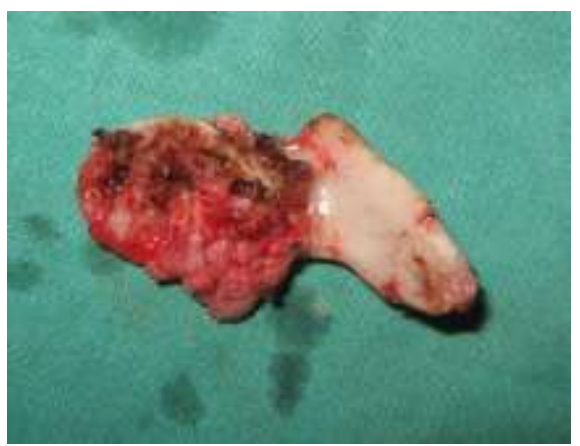

Figure 7: Osseous tissue along with coronoid process of right side

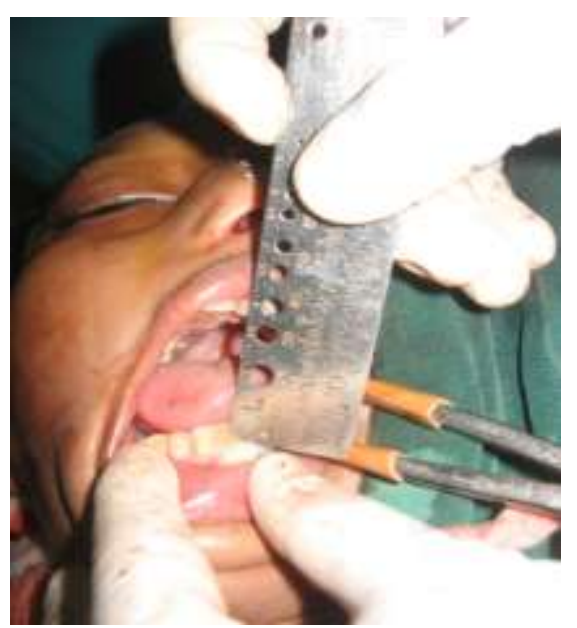

Figure 8: Photograph showing $34 \mathrm{~mm}$ mouth opening achieved during surgery.

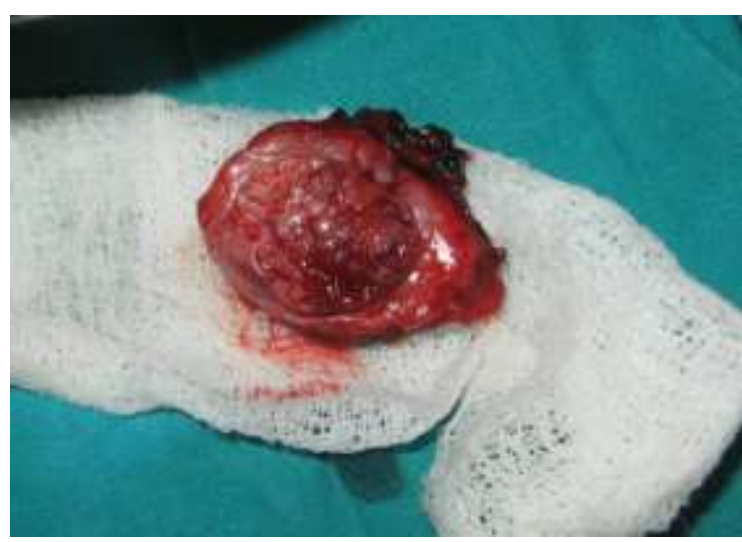

Figure 9: Enucleated cyst along with lining. 


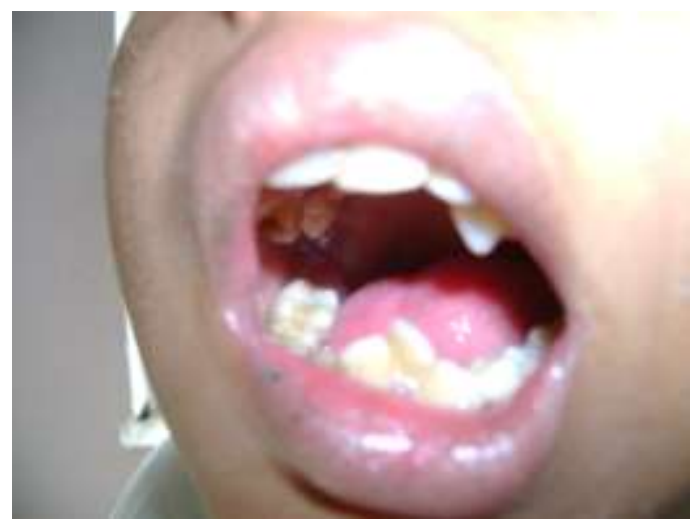

Figure 10: Photograph of the patient after 3 months interval.

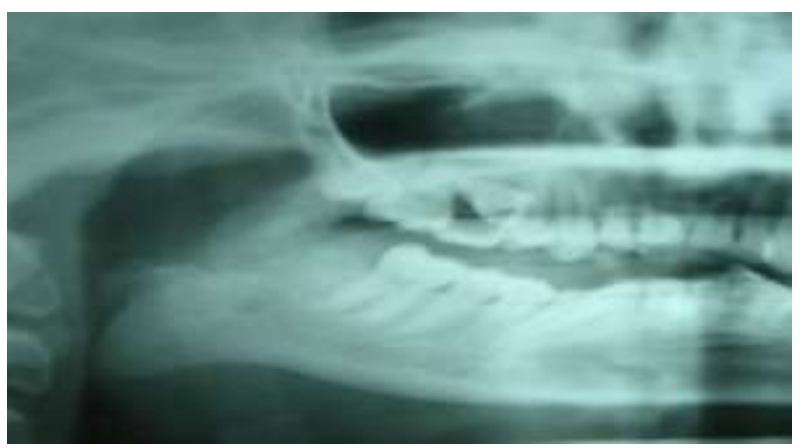

Figure 11: Cropped photograph of orthopantomogram with no evidence of recurrence of OKC on right side after 6 months.

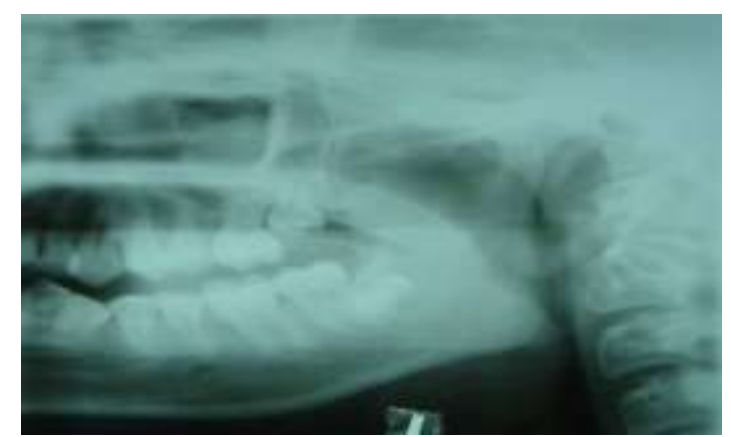

Figure 12: Cropped photograph of orthopantomogram of left side after 6 months with mucous retention cyst in left maxillary antrum and absence of coronoid process.

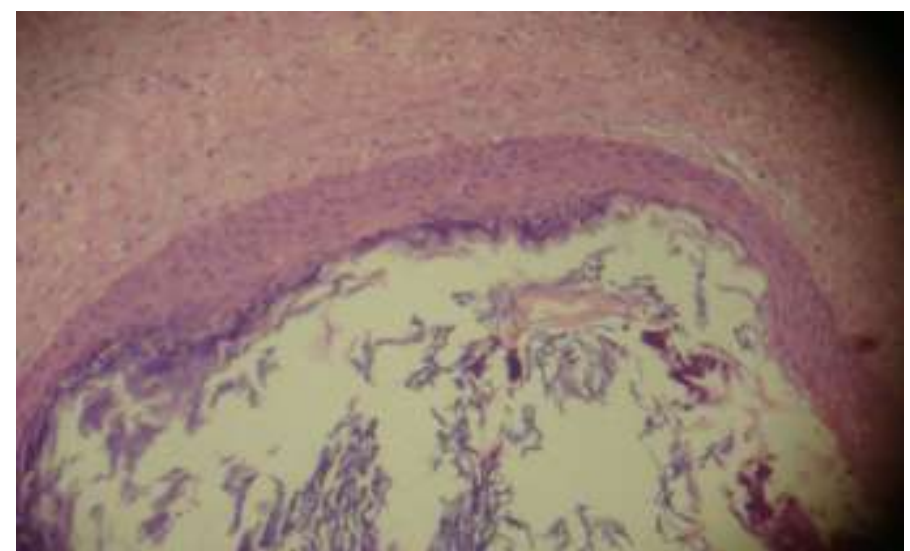

Figure 13: Photomicrograph showing stratified squamous epithelium lining with non polarity of basal cells. This lining is covered with connective tissue wall which consist of collagen fibers, fibroblasts and capillaries. Cystic cavity consists of keratin flecks. 